\title{
Median Neuropathy Caused by Intramuscular Venous Malformation in the Brachialis Muscle at the Elbow: A Case Report
}

\begin{abstract}
Sang-soo Choi $^{1}$, Byung-chul Son ${ }^{1,2}$
${ }^{1}$ Department of Neurosurgery, Seoul St. Mary's Hospital, College of Medicine, The Catholic University of Korea, Seoul, ${ }^{2}$ Catholic Neuroscience Institute, College of Medicine, The Catholic University of Korea, Seoul, Republic of Korea

Corresponding author: Byung-chul Son Department of Neurosurgery, Seoul St. Mary's Hospital, Catholic Neuroscience Institute, College of Medicine, The Catholic University of Korea, 222 Banpo-daero, Seocho-gu, Seoul 06591, Republic of Korea Tel: $+82-2-2258-6122$

Fax: +82-2-594-4248

E-mail: sbc@catholic.ac.kr

Received: May 5, 2020

Venous malformations (VMs) are composed of abnormal collections of veins that have different luminal sizes and wall thicknesses, and geographically can appear superficial, deep, diffuse, localized, or not uncommonly, multiple. Since VMs are a congenital lesion, their occurrence in the late 50 s is rare. In addition, intramuscular VMs comprise an uncommon subgroup of VMs. They are often mistaken for tumors because of a similar presentation. Here we report on a rare intramuscular VM originating from the brachialis muscle in the left arm, which caused a local mass effect, showing the symptoms associated with irritation of the median nerve. The lesion was diagnosed by typical magnetic resonance imaging findings. Surgical resection was chosen for this symptomatic, well-localized lesion in the distal brachialis muscle; it resulted in complete symptomatic relief without recurrence.
\end{abstract}

Revised: June 5, 2020

Accepted: June 24, 2020
Key Words: Brachialis muscle; Elbow; Median neuropathy; Vascular malformation

\section{INTRODUCTION}

Venous malformations (VMs) are a commonly encountered entity in clinical practice, with an estimated incidence of 1 to 2 in 10,000 births and a prevalence of $1 \%{ }^{4,19)}$. VMs are composed of abnormal collections of veins with a variable luminal size and wall thickness, often multiple ${ }^{17)}$. They are often less well-circumscribed than are vascular tumors, such as infantile hemangiomas, and can be interspersed with adipose tissue or within various kinds of atrophic or degenerative muscle ${ }^{13)}$. Although all VMs are present at birth, but they are also identified in adolescence and adulthood ${ }^{13)}$. VMs occur at a frequency of $40 \%$ in the head and neck region, $40 \%$ in the extremities, and $20 \%$ in the trunk ${ }^{2}$. VMs in the extremities often violate surrounding fascial planes and can infiltrate subcutaneous tissue, muscle, bone, joints, neurovascular structures, and even viscera ${ }^{2)}$.

Intramuscular VMs comprise an uncommon subgroup of $\mathrm{VMs}^{15}$. They are often mistaken for tumors, because of a similar presentation and because of improper nomenclature ${ }^{8)}$. Although most intramuscular VMs in the extremities have been reported to present with a growing palpable mass with or without pain ${ }^{10)}$, the development of neurological symptoms is rare. The authors report a rare intramuscular VM originating from the brachialis muscle and showing symptoms of median nerve involvement

\section{CASE REPORT}

A 58-year-old, right-handed male patient presented with a gradually growing mass in the distal upper arm and mild weakness in left-hand grasping. The mass occurred 6 months prior to the presentation, and there was no pain when it first occurred. The mass gradually increased; in the morning, it became larger, and in the afternoon, it became smaller (Fig. 1A). A month previously, when he touched the mass, he felt tenderness and tingling in the thenar area of his left hand. Two weeks later, the mass got bigger, and his left-hand grip weakened; when he grabbed an object, he began to drop it.

On examination, there was no objective weakness in forearm pronation, wrist flexion, flexion of the first 3 digits and thumb opposition, or abduction, which are innervated by the left median nerve, nor any objective sensory disturbance in the radial $2 / 3$ of the palm that is innervated by the palmar cutaneous branch of the median nerve. There was no tenderness in the mass itself, but when the medial of the mass was pressed, there was pain along with tingling instantaneously in the thenar side of the left palm. It was not mobile. X-ray examination showed a small calcification in the soft tissue in front of the 
distal humerus (Fig. 1B). Magnetic resonance imaging (MRI) of the upper extremity revealed a 3-cm-sized, lobulated mass within the brachial muscle in the distal upper arm (Fig. 2B).
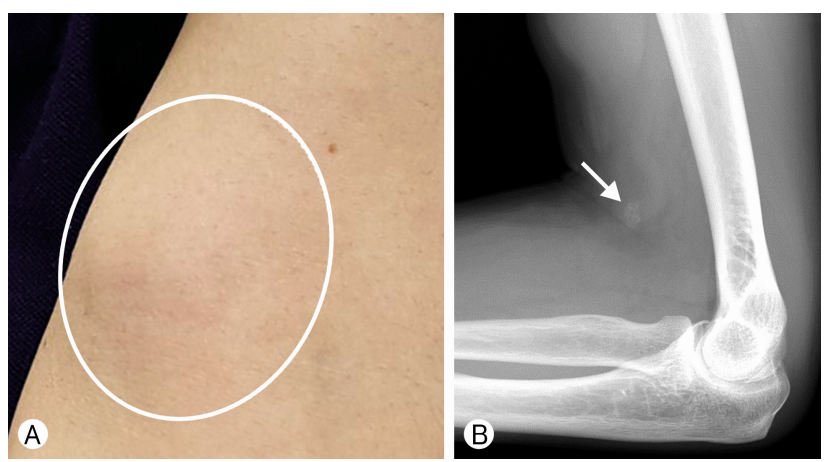

Fig. 1. Presentation of intramuscular venous malformation in the left cubital fossa. (A) Clinical photograph showing a buldging mass in the medial side of the left arm cubital fossa (white circle). (B) On the $\mathrm{X}$-ray, a small round calcification thought to be a phlebolith is seen.
A T2-weighted fast spin-echo image revealed a well-demarcated, lobulated mass with a hyperintense signal (Fig. 2B). Inside the hyperintense lesion, the heterogeneous appearance with low signal intensities indicated hemorrhage or thrombosis, and often dilated vascular structures were identified. In the T1-weighted image, it was isointense with lesion extent appearing inconspicuous to adjacent muscular tissue (Fig. 2C). Gadolinium administration resulted in heterogeneous enhancement within the mass (Fig. 2C). The veins of the antecubital fossa were dilated and showed ectasia. The left median nerve was not directly involved by the lesion, but was displaced by the swollen brachialis muscle (Fig. 2D). With these unique MR findings, the lesion was thought to be a low-flow, VM. Considering the gradual enlargement of the mass and worsening of the pain and neurological symptoms, surgery was planned, and consent was obtained.

After we made a lazy S-shaped vertical incision along the medial antecubital area, we carried dissection down to the antebrachial fascia. After securing the left median nerve, we dissected the swollen brachial muscle under microscopic vision.
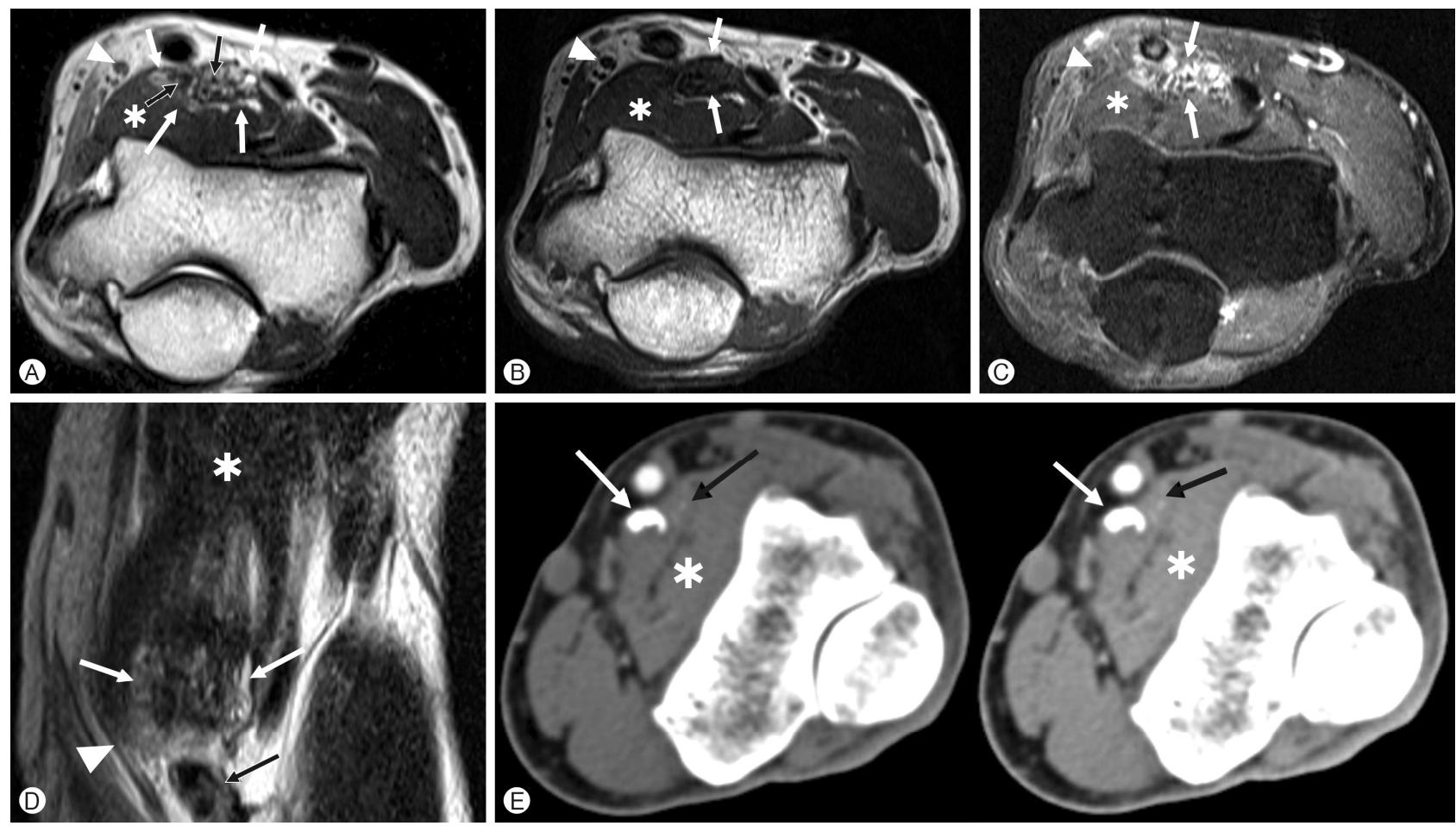

Fig. 2. Magnetic resonance imaging of venous malformation (VM) originating from the brachialis muscle. (A) Axial T2-weighted fast spin echo image reveals hyperintense signal within the VM (white arrows) with excellent definition of lesion extent. Multiple signal voids (black arrows) indicating vascular channels and fibrous septa are noted within the VM, which is located within the brachialis muscle (asterisk), and the median nerve (white arrowhead) is not involved directly. (B) Axial T1-weighted image revealing isointense VM with lesion extent (white arrows). Multiple signal voids are seen. The brachialis muscle (asterisk) and the median nerve (white arrowhead) are identified. (C) Gadolinium-enhanced fat-saturated T1 image reveals heterogeneously enhanced VM (white arrows). (D) Coronal T2-weighted image shows the extent of lesion (white arrows) within the brachialis muscle (asterisk). The left median nerve (white arrow head) is displaced medially. The draining vein (black arrow) within the cubital fossa is dilated. (E) Axial images of the computed tomographic scan (left) with enhancement (right) of the VM within the brachialis muscle (asterisk). The lesion was isodense and not well demarcated from the brachialis muscle. No obvious enhancement was found in this VM (right image). A large calcification (white arrow) and small spotted, calcific density indicating calcific fibrous septa (black arrow) are noted within the VM. 

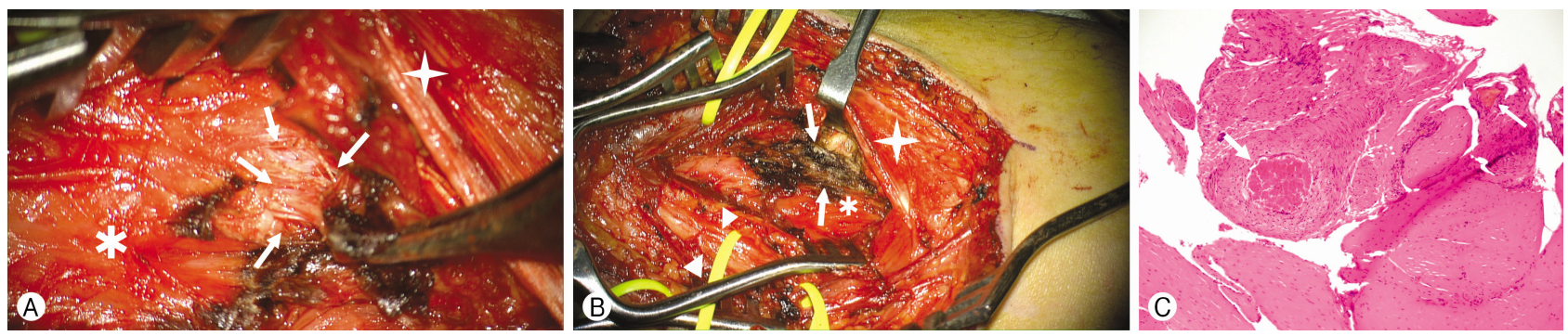

Fig. 3. Intraoperative findings and histologic examinations of intramuscular venous malformation (VM). (A) An intraoperative photograph showing multiple dilated venous channels and fibrous septa (white arrows) within the VM. The VM are located within the brachialis muscle (asterisk). The biceps muscle (white star) is retracted for exposure. (B) An intraoperative photograph taken after excision of the VM. The hemoclips are used for hemostasis and excision. The left median nerve (white arrowhead) and the biceps muscle (white star) are identified. (C) A histologic photograph showing various-sized dilated vessels (arrows) (hematoxylin and eosin stain [H\&E], $\times 25$ magnification). No cellular atypia are noted.

We did circumferential dissection by identifying a fairly firm venous structure (Fig. 3A). Between the muscle fibers, multiple dilated large venous channels and fibrous septa were identified and ligated with hemoclips. Bleeding venous structures and dilated drainers were controlled by bipolar coagulation. The VM was firmly attached to the surrounding brachialis muscle. However, no difficulty was found in complete excision (Fig. 3B, C). No neurological abnormalities were observed after complete excision, and the mass did not appear again. By one year after surgery, the mass was no longer present, and there was no abnormality in the left hand and arm movements and sensations.

\section{DISCUSSION}

\section{Intramuscular VM}

Identification and classification of vascular anomalies were hampered historically by the use of confusing nomenclature ${ }^{7)}$. Early classification suggested by Virchow ${ }^{20)}$ and Wegener ${ }^{21)}$ classified vascular lesions according to the pathologic appearance of the vessel ${ }^{7)}$. Vascular growths were divided into angiomas and lymphangiomas without consideration of the biologic behavior and natural history of the vascular lesions ${ }^{7}$. Consequently, there was a tendency to identify any vascular anomaly as a hemangioma ${ }^{5-7,13,16)}$. A variety of terms including "venous angioma”, "cavernous angioma”, "cavernous hemangioma", and "phlebangioma" have been used in the medical literature to describe these anomalies ${ }^{5,7)}$. These terms have led to confusion with the more common proliferating or true hemangiomas of infancy ${ }^{5,7)}$. For example, capillary hemangioma, nevus flammeus, and port-wine stain have all been used in the literature to describe a capillary malformation of the skin ${ }^{16}$.

In 1982, Mulliken and Glowacki ${ }^{16)}$ proposed a modern classification of vascular anomalies according to the lesion's biologic and pathologic differences; all vascular anomalies were assigned to 1 of 2 broad categories: hemangiomas and vascular
Table 1. Classification of vascular anomalies according to vascular dynamics

\begin{tabular}{|c|c|}
\hline 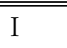 & Hemangioma \\
\hline II & Vascular malformations \\
\hline A & Low-flow (venous malformation) \\
\hline B & High-flow (arteriovenous malformation) \\
\hline
\end{tabular}

[Reprinted from "Hemangiomas, vascular malformations, and lymphovenous malformations: classification and methods of treatment.", by Jackson IT, et al., 1993, Plast Reconstr Surg, 91, pp. 1216-1230. Copyright 1993 by the Lippincott Williams \& Wilkins. Reprinted with permission].

malformations. The former category was later expanded to include vascular tumors. The suffix "-oma” was to be reserved for only those lesions exhibiting increased cellular turnover, the classic example within this category being the infantile hemangioma ${ }^{5,13)}$. The term "vascular malformation" was applied to those lesions present at birth growing commen-surately or pari passu with the child ${ }^{13)}$. The vascular malformations were composed of normal "mature" flat endothelial-lined vascular spaces with a normal rate of cell turnover and were further subdivided into capillary malformations, VMs, arterial (arterio venous) malformations, and lymphatic malformations ${ }^{5,13)}$. In

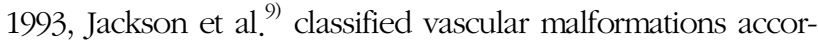
ding to flow patterns instead of the former anatomicopathological classification for ease of investigation and treatment (Table 1). They simplified flow patterns within vascular malformations as either low-flow (VMs) or high-flow (arteriovenous malformations), keeping separate categories for lymphangiomas and hemangiomas, with the purpose of creating "system directly related to investigation and treatment"9).

\section{Clinical Manifestation and Diagnosis}

The diagnosis of VM and differentiation from other vascular malformations can be usually made by clinical history and 
physical examination ${ }^{13)}$. Because VMs are congenital lesions, they may be identified at birth. However, they are usually identified from infancy to puberty, which is the period of greatest enlargement of the lesion ${ }^{7}$. Continued linear growth within the malformation despite the end of somatic growth in late adolescence often results in clinical manifestations later in life and is typically the case in deeper lesions ${ }^{20)}$.

VMs typically appear as soft, compressible, blue-tinged masses that can enlarge with dependent position and Valsalva maneuver ${ }^{1)}$. The blue tinge is considered pathognomonic and is caused by dilated venous channels within the dermis ${ }^{6}$. Forty percent of VMs occur in the head and neck region, and may involve the mucosa of the tongue, palate, orbital, mandibular, or neck region, even direct involvement of the ophthalmic branch of the trigeminal nerve in the face ${ }^{2,11)}$. VMs in the extremities often infiltrate the surrounding tissues ${ }^{13)}$. As such, patients commonly experience symptoms as a result of several mechanisms. Venous engorgement secondary to dependent positioning, exercise, after prolonged stasis, or after morning awakening frequently results in significant swelling and pain ${ }^{13)}$. Mass effect may cause local compression of the nerve and muscular contracture or restricted range of motion of an adjacent joint ${ }^{13)}$. Local hemorrhage and local stasis on a background of chronic low-grade intravascular coagulopathy thromboembolic state within the lesion can occur ${ }^{13,14)}$. In the current case, a VM occurred in late adulthood at the age of 58 and originated from the brachialis muscle. It showed swelling in the morning due to venous stasis and symptoms of left median nerve irritation due to local mass effect.

MRI is the imaging modality of choice for $\mathrm{VMs}^{13)}$, which classically appear as either isointense or hypointense on the T1-weighted sequences ${ }^{12,13)}$. The lesions appear focal, diffuse, or demonstrate lobulated margins. A more heterogeneous appearance can be identified in the setting of hemorrhage or thrombosis, and often dilated and serpiginous vascular structures can be identified compatible with abnormal veins ${ }^{13)}$. Lower signal area or signal voids may represent dystrophic calcification or phlebolith on all imaging sequences ${ }^{12)}$. In addition to calcification, lower signal areas on $\mathrm{T} 2$ can be caused by either vascular channels or fibrofatty septa ${ }^{12)}$. Gadolinium administration results in homogeneous or heterogeneous enhancement within the substrate of a $\mathrm{VM}^{13)}$.

\section{Treatment of Intramuscular VM}

Treatment is generally indicated if the lesion causes pain, functional impairment, or aesthetic problems, as in craniofacial lesions ${ }^{2}$. With the exception of some superficial VMs where laser therapy is effective, VMs are generally treated with direct surgery and sclerotherapy ${ }^{13)}$. Surgical resection is considered preferable if the lesion could be completely removed so as to avoid recurrence ${ }^{8)}$. This includes patients with focal well-defined VMs that are thrombosed, confined to a single or spe- cialized muscle group, or causing a neurological or compression syndrome, and patients where there is a good possibility of anatomical and functional restoration ${ }^{8)}$. Many lesions are infiltrative, however, and involve multiple muscle groups or fascial planes where surgical resection results in an unacceptably high functional and cosmetical deficit ${ }^{18)}$. Sclerotherapy has been increasingly incorporated in surgical regimens and now has been considered as an adjunct to surgery or the stand-alone therapy of choice for most $\mathrm{VMs}^{3,13,18)}$. We chose surgical resection in the current case because the lesion was well localized within the brachialis muscle and caused local neurological symptoms by mass effect. The treatment resulted in complete relief of the symptoms of VM, with no recurrence up to 2 years after surgery.

\section{CONCLUSION}

Here, we report on a rare intramuscular VM originating from the brachialis muscle in the left arm, which caused the local mass effect, showing the symptoms associated with irritation of the median nerve. The lesion was diagnosed through a typical MR imaging finding. Because the lesion was well-localized within the brachialis muscle, surgical resection resulted in complete symptomatic relief without recurrence.

\section{CONFLICTS OF INTEREST}

No potential conflict of interest relevant to this article was reported.

\section{REFERENCES}

1. Abernethy LJ: Classification and imaging of vascular malformations in children. Eur Radiol 13:2483-2497, 2003

2. Dubois J, Soulez G, Oliva VL, Berthiaume MJ, Lapierre C, Therasse E: Soft-tissue venous malformations in adult patients: imaging and therapeutic issues. Radiographics 21:1519-1531, 2001

3. Dubois JM, Sebag GH, De Prost Y, Teillac D, Chretien B, Brunelle FO: Soft-tissue venous malformations in children: percutaneous sclerotherapy with Ethibloc. Radiology 180:195-198, 1991

4. Eifert S, Villavicencio JL, Kao TC, Taute BM, Rich NM: Prevalence of deep venous anomalies in congenital vascular malformations of venous predominance. J Vasc Surg 31:462-471, 2000

5. Enjolras O: Classification and management of the various superficial vascular anomalies: hemangiomas and vascular malformations. J Dermatol 24:701-710, 1997

6. Enjolras O, Ciabrini D, Mazoyer E, Laurian C, Herbreteau D: Extensive pure venous malformations in the upper or lower limb: a review of 27 cases. J Am Acad Dermatol 36:219-225, 1997

7. Garzon MC, Huang JT, Enjolras O, Frieden IJ: Vascular malformations: Part I. J Am Acad Dermatol 56:353-370, 2007

8. Hein KD, Mulliken JB, Kozakewich HP, Upton J, Burrows PE: 
Venous malformations of skeletal muscle. Plast Reconstr Surg 110:1625-1635, 2002

9. Jackson IT, Carreño R, Potparic Z, Hussain K: Hemangiomas, vascular malformations, and lymphovenous malformations: classification and methods of treatment. Plast Reconstr Surg 91: 1216-1230, 1993

10. Kim EY, Ahn JM, Yoon HK, Suh YL, Do YS, Kim SH, et al.: Intramuscular vascular malformations of an extremity: findings on MR imaging and pathologic correlation. Skeletal Radiol 28: 515-521, 1999

11. Kim JH, Choi JG, Son BC: Venous malformation (cavernous hemangioma) of the supraorbital nerve. Asian J Neurosurg 13: 499-502, 2018

12. Konez O, Burrows PE: Magnetic resonance of vascular anomalies. Magn Reson Imaging Clin N Am 10:363-388, vii, 2002

13. Legiehn GM, Heran MK: Venous malformations: classification, development, diagnosis, and interventional radiologic management. Radiol Clin North Am 46:545-597, vi, 2008

14. Mazoyer E, Enjolras O, Laurian C, Houdart E, Drouet L: Coagulation abnormalities associated with extensive venous malformations of the limbs: differentiation from Kasabach-Merritt synd- rome. Clin Lab Haematol 24:243-251, 2002

15. Muchemwa FC, Ishihara T, Matsushita S: Intramuscular venous malformation in the upper arm with gross calcifications and compression of the ulnar nerve. Scand J Plast Reconstr Surg Hand Surg 41:93-95, 2007

16. Mulliken JB, Glowacki J: Hemangiomas and vascular malformations in infants and children: a classification based on endothelial characteristics. Plast Reconstr Surg 69:412-422, 1982

17. North PE, Mihm MC, Jr.: Histopathological diagnosis of infantile hemangiomas and vascular malformations. Facial Plast Surg Clin North Am 9:505-524, 2001

18. Puig S, Casati B, Staudenherz A, Paya K: Vascular low-flow malformations in children: current concepts for classification, diagnosis and therapy. Eur J Radiol 53:35-45, 2005

19. Vikkula M, Boon LM, Mulliken JB: Molecular genetics of vascular malformations. Matrix Biol 20:327-335, 2001

20. Virchow R: Angioma in die krankhaften Geschwulste. Berlin, DE: Hirschwald, 1863

21. Wegener G: Ueber lyymphangiome. Arch Klin Chir 20:641-707, 1977 